Archive for

Organic Chemistry

Arkivoc 2019, part vi, 149-157

\title{
Formation of zwitterionic salts via three-component reactions of barbituric/thiobarbituric acid, N-heterocyclic compounds and dialkyl acetylenedicarboxylates
}

\section{Mohammad Anary-Abbasinejad* and Fereshteh Nejad-Shahrokhabadi}

Department of Chemistry, Faculty of Science, Vali-e-Asr University of Rafsanjan, Rafsanjan 77176, Iran

E-mail: m.anary@vru.ac.ir

Received 07-11-2019

Accepted 09-12-2019

Published on line 10-26-2019

\section{Abstract}

A simple and efficient one-pot three-component reaction between $\mathrm{N}$-heterocycles and dialkyl acetylenedicarboxylates in the presence barbituric/thiobarbituric acid has been studied. In all cases, new and stable 1,4-diionic compounds were obtained in excellent yields.

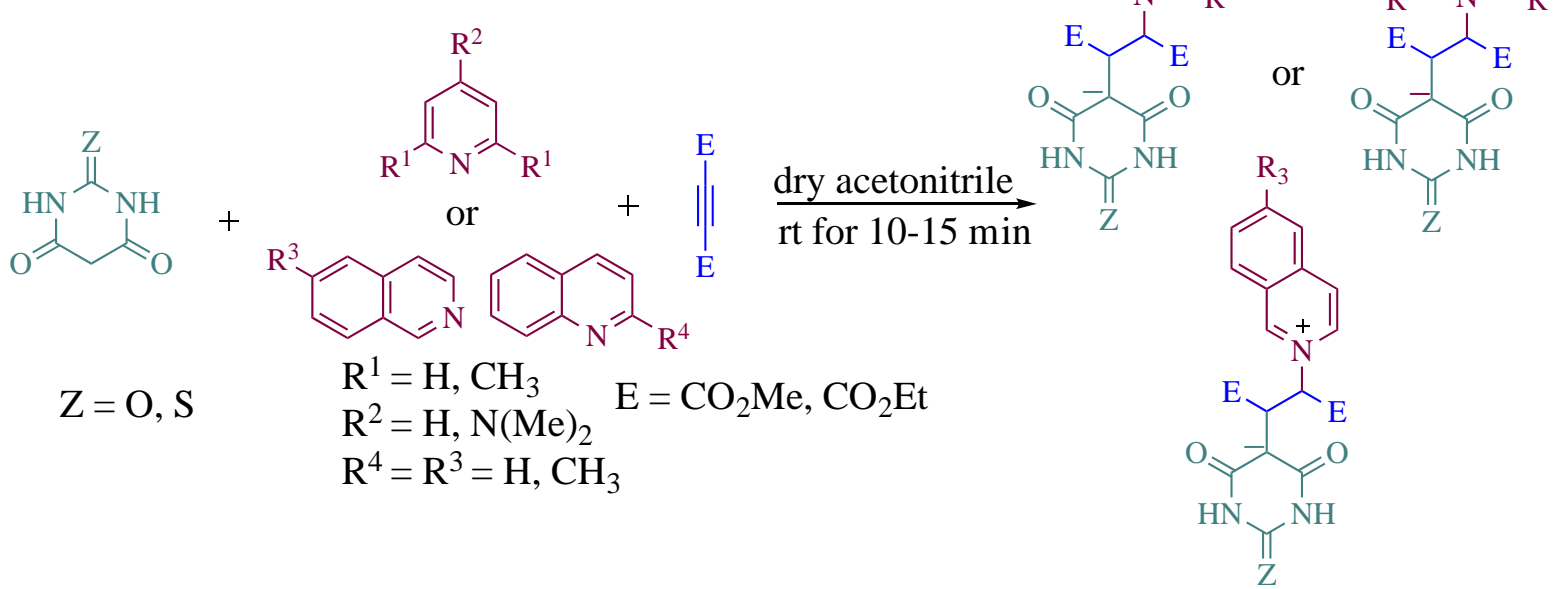

Keywords: Zwitterion, N-heterocycles, dialkyl acetylenedicarboxylate, barbituric acid 


\section{Introduction}

Barbituric and 2-thiobarbituric acids are an important class of organic compounds which constitute the main skeleton of a series of barbiturate/thiobarbiturate drugs used as hypnotics, antioxidants, sedatives, anticonvulsants, anaesthetics, antifungal, and central nervous system depressants. ${ }^{1-3}$ Barbiturates have also found wide applications for the manufacture of plastics, ${ }^{4}$ textiles, ${ }^{5}$ and polymers. ${ }^{6}$

$\mathrm{N}$-Heterocyclic zwitterions are reactive species that are widely used in organic synthesis, pharmaceuticals and bioinorganic chemistry. ${ }^{7-9}$ Pyridine zwitterions are usually synthesized via the addition of a pyridine derivative to a reactive double bond or a carbene. ${ }^{10-12}$ Such compounds have also been prepared by the reaction of nitrogen aromatic heterocycles with acetylene diesters in the presence of strong $\mathrm{CH}$-acidic organic compounds such as 1,3-dicarbonyl compounds. ${ }^{13-16}$ There are also some recent reports on the synthesis of $\mathrm{N}$ heterocyclic zwitterions by multi-component reactions. ${ }^{17-20}$

Here we report a new three-component reaction of pyridine, quinoline or isoquinoline derivatives with dialkyl acetylenedicarboxylate (DAAD) derivatives in the presence of barbituric or thiobarboturic acid for the synthesis of new functionalized $N$-heterocycle zwitterions.

\section{Results and Discussion}

We first studied the reaction between quinoline $\mathbf{1 a}$, thiobarbituric acid $\mathbf{2 a}$ and dimethyl acetylenedicarboxylate (DMAD) $\mathbf{3 a}$ in dry acetonitrile as solvent (Scheme 1). When DMAD was added to a mixture of quinoline and thiobarbituric acid in dry acetonitrile at $25^{\circ} \mathrm{C}$, a smooth reaction proceeded and after $10 \mathrm{~min}$ a solid was separated from the reaction mixture. Washing the solid with acetonitrile afforded zwitterionic salt $4 a$ in $97 \%$ yield.

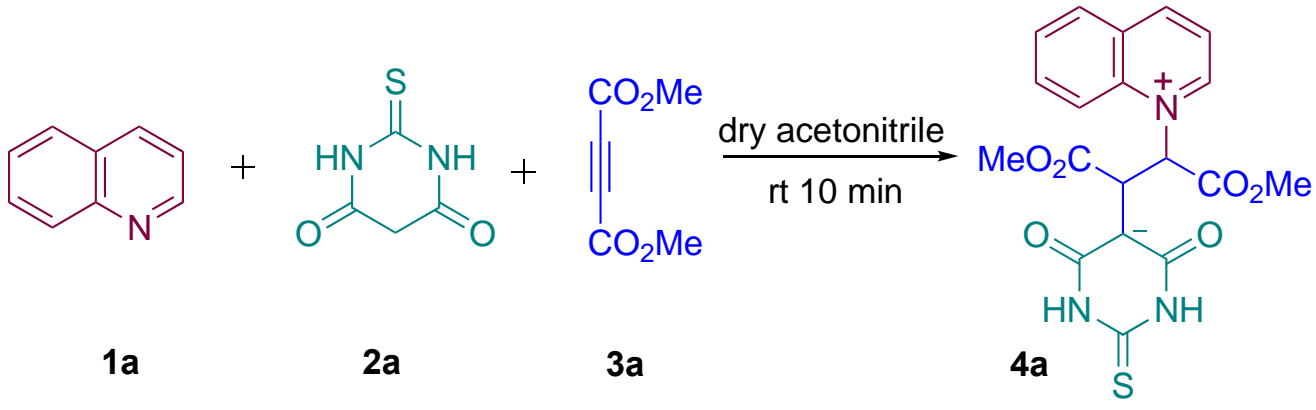

Scheme 1. Synthesis of quinolinium zwitterion $\mathbf{4 a .}$

To define the scope and generality of the method, a series of $\mathrm{N}$-heterocycles, including pyridine, quinolone and isoquinoline derivatives were reacted with DMAD or diethyl acetylenedicarboxylate (DEAD) in the presence of barbituric or thiobarbituric acid and in each case the related $\mathrm{N}$-heterocyclic zwitterion was obtained in high yield (Table 2).

All the synthesized compounds $4 a-I$ were unknown and were identified by CHNS analysis and IR, ${ }^{1} \mathrm{H}$ and ${ }^{13} \mathrm{C}$ NMR spectroscopic data. For example the 500-MHz ${ }^{1} \mathrm{H}$ NMR spectrum of compound 4 a exhibited two sharp signal at 3.59 and 3.78 ppm for methyl groups of the DMAD unit and two sharp doublet signals at 4.33 and $4.81 \mathrm{ppm}$ for two methine groups. Aromatic hydrogens gave rise to characteristic signals in the aromatic region of the spectrum and two singlet signals were observed for two NH protons at 11.72 and $11.86 \mathrm{ppm}$. The 
observation of two NH groups in different chemical shifts shows that the rotation of the barbituric acid ring around the ${ }^{-} \mathrm{C}-\mathrm{CH}$ bond is restricted related to the attractive interaction between positive and negative parts of the molecule. A similar interaction has been reported for phosphorus 1,4-diionic compounds. ${ }^{21}$ The ${ }^{1} \mathrm{H}$ decoupled ${ }^{13} \mathrm{C}$ NMR spectrum of 4 a showed fourteen distinct resonances in agreement with the suggested structure. The carbons of the thionyl $(C=S)$ and carbonyl $(C=O)$ groups resonated at 178.1, 172.5, 169.6 and 165.2 ppm, respectively.

Compounds 4a-I possess two stereogenic centers and may exist as two diastereomers. The NMR spectra showed that compounds $\mathbf{4 a - e}$ and $\mathbf{4 g}$ were obtained as only one stereoisomer, but the other products were isolated as a mixture of two isomers. However we could not find a general trend for the stereoselectivity of the reaction. It was also notable that in the case of 2,6-dimethylpyridinium salts the rotation of the pyridine ring around the $\mathrm{N}^{+}-\mathrm{C}$ bond is restricted and the NMR spectra showed distinct signals for the ring ortho and meta $\mathrm{CH}$ groups and for the methyl groups on the pyridine ring.

Table 1. Scope of the reaction for synthesis of N-heterocyclic zwitterions $4 a-I$

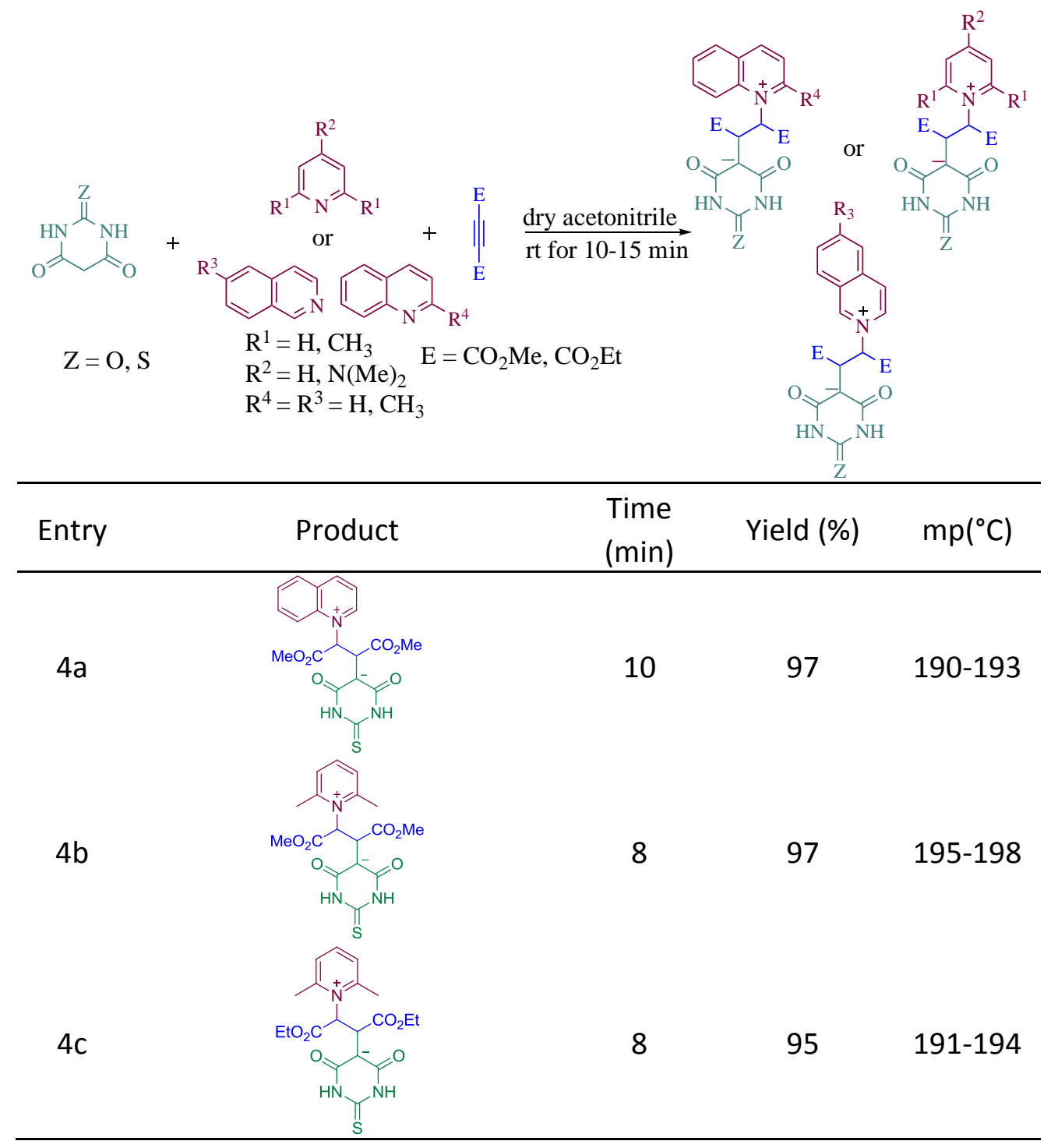


Table 1. Continued

\begin{tabular}{|c|c|c|c|c|}
\hline Entry & Product & $\begin{array}{l}\text { Time } \\
\text { (min) }\end{array}$ & Yield (\%) & $\mathrm{mp}\left({ }^{\circ} \mathrm{C}\right)$ \\
\hline $4 d$ & & 10 & 90 & $278-280$ \\
\hline 40 & & 10 & 95 & $177-180$ \\
\hline$\Delta f$ & & 15 & 97 & 197-199 \\
\hline $4 g$ & & 10 & 96 & $187-190$ \\
\hline $4 \mathrm{~h}$ & & 7 & 97 & $194-196$ \\
\hline $4 i$ & & 15 & 97 & $201-203$ \\
\hline $4 j$ & & 8 & 98 & $210-211$ \\
\hline $4 k$ & & 8 & 94 & $197-200$ \\
\hline 41 & & 10 & 92 & $190-193$ \\
\hline
\end{tabular}

Mechanistically, the formation of zwitterion $4 a$ is shown in Scheme 2 using quinoline as an example. The nucleophilic addition of quinoline to DMAD as a Michael acceptor generates the salt I as an intermediate. 
Intermediate I is protonated by thiobarbituric acid. Then, the positively charged ion II is attacked by the conjugate base of thiobarbituric acid to create pyridinium ylide III which converts to zwitterion 4a by a 1,3proton transfer.

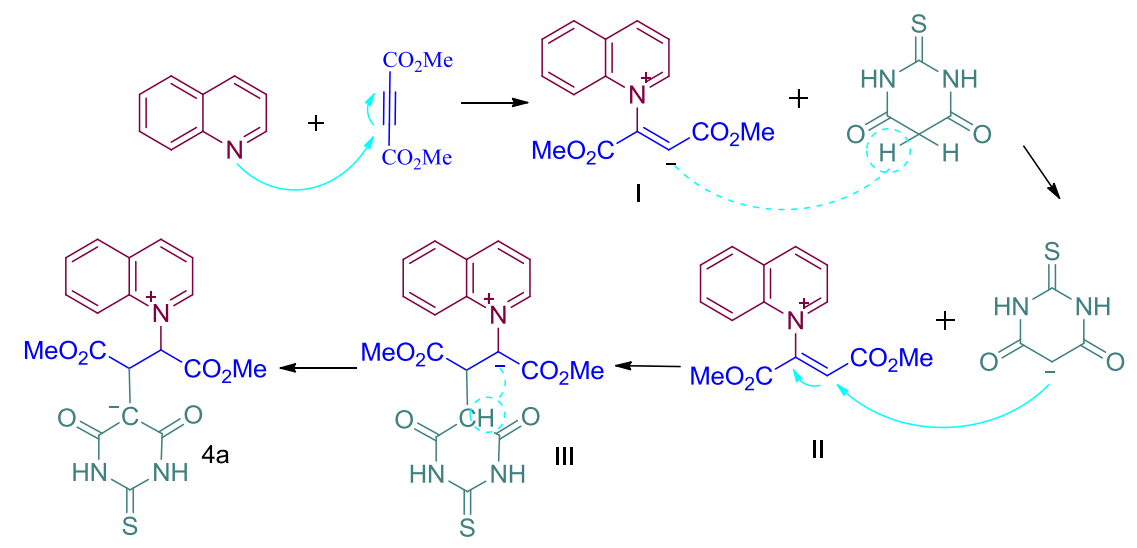

Scheme 2. A possible mechanism for the synthesis of zwitterion $4 a$

\section{Conclusions}

We report herein a simple and efficient one-pot three-component reaction between $\mathrm{N}$-heterocycles, dialkyl acetylenedicarboxylates and barbituric or thiobarbituric acid for the synthesis of new N-heterocyclic zwitterions in good yields. The advantages of the method are readily available starting materials, easy purification of products and simple and neutral reaction conditions.

\section{Experimental Section}

General. All chemicals and solvents were purchased from commercial sources and used without further purification. Melting points were determined by a Bamslead Electrothermal 9200 apparatus. ${ }^{1} \mathrm{H} N M R$ and ${ }^{13} \mathrm{C}$ NMR spectra were recorded on a Varian model UNITY Inova $500 \mathrm{MHz}$ spectrometer in DMSO- $d_{6}$ as solvent. Data are reported in parts per million (ppm), from tetramethylsilane (TMS) as an internal standard in DMSO$d_{6}$. IR spectra were recorded on a Shimadzu IR-470 spectrometer, and only major peaks are reported in $\mathrm{cm}^{-1}$.

General procedurefor the synthesis of zwitterionic salts $4 a-I$ is exemplified by the synthesis of zwitterionic salt 4a. To a mixture of quinoline $(1 \mathrm{mmol})$ and thiobarbituric acid $(1 \mathrm{mmol})$ in $\mathrm{MeCN}(10.0 \mathrm{~mL}) \mathrm{was}$ added dimethyl acetylenedicarboxylate $(1 \mathrm{mmol})$ and the solution was stirred at $\mathrm{rt}$ for $10 \mathrm{~min}$. The resulting precipitate was filtered off and washed with $\mathrm{MeCN}(10.0 \mathrm{~mL})$ to give a pure yellow solid for analysis.

\section{5-(1,4-Dimethoxy-1,4-dioxo-3-(quinolin-1-ium-1-yl)butan-2-yl)-4,6-dioxo-2-thioxohexahydropyrimidin-5-ide} (4a). Orange solid, mp 190-193 ${ }^{\circ} \mathrm{C}$ (97\% Yield). IR (KBr) $v_{\max } / \mathrm{cm}^{-1}: 3430,3133,1739,1694 .{ }^{1} \mathrm{H} \mathrm{NMR}(500 \mathrm{MHz}$ DMSO-d $d_{6} \delta$ (ppm): $11.86(1 \mathrm{H}, \mathrm{bs}, \mathrm{NH}), 11.72(1 \mathrm{H}, \mathrm{bs}, \mathrm{NH}), 7.04\left(1 \mathrm{H}, \mathrm{td},{ }^{3} \mathrm{~J}_{\mathrm{HH}} 7.8,1.6 \mathrm{~Hz}, \mathrm{H}-\mathrm{Ar}\right), 6.86\left(1 \mathrm{H}, \mathrm{dd},{ }^{3} \mathrm{~J}_{\mathrm{HH}}\right.$ 7.4, $1.6 \mathrm{~Hz}, \mathrm{H}-\mathrm{Ar}), 6.59-6.54(2 \mathrm{H}, \mathrm{m}, \mathrm{H}-\mathrm{Ar}), 6.52\left(1 \mathrm{H}, \mathrm{d},{ }^{3} \mathrm{~J}_{\mathrm{HH}} 8.2 \mathrm{~Hz}, \mathrm{H}-\mathrm{Ar}\right), 5.58\left(1 \mathrm{H}, \mathrm{dd},{ }^{3} J_{\mathrm{HH}} 10.1,3.3 \mathrm{~Hz}, \mathrm{H}-\mathrm{Ar}\right)$, $5.18\left(1 \mathrm{H}, \mathrm{dd},{ }^{3} \mathrm{~J}_{\mathrm{HH}} 3.3,1.5 \mathrm{~Hz}, \mathrm{H}-\mathrm{Ar}\right), 4.81\left(1 \mathrm{H}, \mathrm{d},{ }^{3} \mathrm{~J}_{\mathrm{HH}} 7.2 \mathrm{~Hz}, \mathrm{CH}\right), 4.33\left(1 \mathrm{H}, \mathrm{d},{ }^{3} \mathrm{~J}_{\mathrm{HH}} 7.2 \mathrm{~Hz}, \mathrm{CH}\right), 3.78(1 \mathrm{H}, \mathrm{s}, \mathrm{CH})$, $3.59\left(1 \mathrm{H}, \mathrm{s}, \mathrm{CH}_{3}\right) \cdot{ }^{13} \mathrm{C}$ NMR $\{1 \mathrm{H}\}(125 \mathrm{MHz}$ DMSO-d $) \delta(\mathrm{ppm}): 178.1(\mathrm{C}=\mathrm{S}), 172.5(\mathrm{C}=0), 169.6(\mathrm{C}=0), 165.2$ 
(C=O), 150.2, 143.1, 130.6, 130.1, 127.9, 118.6, 118.5, 115.6, 109.9, 72.4, 65.3, 64.5, 53.4, 51.5. Anal. Calcd for $\left(\mathrm{C}_{19} \mathrm{H}_{17} \mathrm{~N}_{3} \mathrm{O}_{6} \mathrm{~S}\right): \mathrm{C}, 54.93 ; \mathrm{H}, 4.12 ; \mathrm{N}, 10.12 ; \mathrm{S}, 7.72 \%$. Found: $\mathrm{C}, 54.69 ; \mathrm{H}, 4.30 ; \mathrm{N}, 10.25 ; \mathrm{S}, 7.52 \%$.

5-(3-(2,6-Dimethylpyridin-1-ium-1-yl)-1,4-dimethoxy-1,4-dioxobutan-2-yl)-4,6-dioxo-2-thioxohexahydro pyrimidin-5-ide (4b). Yellow solid, mp 195-198 ${ }^{\circ} \mathrm{C}$ (97\% Yield). IR (KBr) $v_{\max } / \mathrm{cm}^{-1}: 3419,2263,1655 .{ }^{1} \mathrm{H} N M R$ (500 MHz DMSO-d $\left.d_{6}\right) \delta(\mathrm{ppm}): 10.62(1 \mathrm{H}, \mathrm{s}, \mathrm{NH}), 10.39(1 \mathrm{H}, \mathrm{s}, \mathrm{NH}), 8.31\left(1 \mathrm{H}, \mathrm{t},{ }^{3} \mathrm{~J}_{\mathrm{HH}} 8.0 \mathrm{~Hz}, \mathrm{H}-\mathrm{Ar}\right), 7.82(1 \mathrm{H}, \mathrm{d}$, $\left.{ }^{3} J_{\mathrm{HH}} 8.0 \mathrm{~Hz}, \mathrm{H}-\mathrm{Ar}\right), 7.79\left(1 \mathrm{H}, \mathrm{d},{ }^{3} J_{\mathrm{HH}} 8.0 \mathrm{~Hz}, \mathrm{H}-\mathrm{Ar}\right), 6.87\left(1 \mathrm{H}, \mathrm{d},{ }^{3} J_{\mathrm{HH}} 8.1 \mathrm{~Hz}, \mathrm{CH}\right), 4.79\left(1 \mathrm{H}, \mathrm{d},{ }^{3} J_{\mathrm{HH}} 8.1 \mathrm{~Hz}, \mathrm{CH}\right), 3.75$ $\left(3 \mathrm{H}, \mathrm{s}, \mathrm{CH}_{3}\right), 3.58\left(3 \mathrm{H}, \mathrm{s}, \mathrm{CH}_{3}\right), 3.09\left(3 \mathrm{H}, \mathrm{s}, \mathrm{CH}_{3}\right), 2.60\left(3 \mathrm{H}, \mathrm{s}, \mathrm{CH}_{3}\right) \cdot{ }^{13} \mathrm{C} \mathrm{NMR}\{1 \mathrm{H}\}\left(125 \mathrm{MHz} \mathrm{DMSO}-d_{6}\right) \delta(\mathrm{ppm}):$ $174.2(\mathrm{C}=\mathrm{S}), 173.5(\mathrm{C}=\mathrm{O}), 173.07(\mathrm{C}=\mathrm{O}), 168.0(\mathrm{C}=\mathrm{O}), 159.5,158.3,146.2,128.2,127.7,83.5,64.6,54.5,52.8$, 40.9, 22.4, 21.3. Anal. Calcd for $\left(\mathrm{C}_{17} \mathrm{H}_{19} \mathrm{~N}_{3} \mathrm{O}_{6} \mathrm{~S}\right): \mathrm{C}, 51.90 ; \mathrm{H}, 4.87 ; \mathrm{N}, 10.68 ; \mathrm{S}, 8.15 \%$. Found: $\mathrm{C}, 51.72 ; \mathrm{H}, 4.68 ; \mathrm{N}$, $10.51 ; \mathrm{S}, 8.21 \%$.

5-(3-(2,6-Dimethylpyridin-1-ium-1-yl)-1,4-diethoxy-1,4-dioxobutan-2-yl)-4,6-dioxo-2-thioxohexahydropyrimidin-5-ide (4c). Yellow solid, mp 191-194 ${ }^{\circ} \mathrm{C}\left(95 \%\right.$ Yield). ${ }^{1} \mathrm{H}$ NMR (500 MHz DMSO- $\left.d_{6}\right) \delta(p p m): 10.64(1 \mathrm{H}$, $\mathrm{s}, \mathrm{NH}), 10.42(1 \mathrm{H}, \mathrm{s}, \mathrm{NH}), 8.32\left(1 \mathrm{H}, \mathrm{t},{ }^{3} \mathrm{~J}_{\mathrm{HH}} 7.9 \mathrm{~Hz}, \mathrm{H}-\mathrm{Ar}\right), 7.86-7.78(2 \mathrm{H}, \mathrm{m}, \mathrm{H}-\mathrm{Ar}), 6.88\left(1 \mathrm{H}, \mathrm{d},{ }^{3} \mathrm{JHH}_{\mathrm{HH}} 8.4 \mathrm{~Hz}, \mathrm{CH}\right)$, $4.77\left(1 \mathrm{H}, \mathrm{d},{ }^{3} \mathrm{~J}_{\mathrm{HH}} 8.4 \mathrm{~Hz}, \mathrm{CH}\right), 4.24\left(2 \mathrm{H}, \mathrm{q},{ }^{3} \mathrm{~J}_{\mathrm{HH}} 7.0 \mathrm{~Hz}, \mathrm{CH}_{2}\right), 4.15-4.01\left(2 \mathrm{H}, \mathrm{m}, \mathrm{CH}_{2}\right), 3.10\left(3 \mathrm{H}, \mathrm{s}, \mathrm{CH}_{3}\right), 2.66(3 \mathrm{H}, \mathrm{s}$, $\left.\mathrm{CH}_{3}\right), 1.14\left(3 \mathrm{H}, \mathrm{t},{ }^{3} \mathrm{~J}_{\mathrm{HH}} 7.0 \mathrm{~Hz}, \mathrm{CH}_{3}\right), 1.12\left(3 \mathrm{H}, \mathrm{t},{ }^{3} \mathrm{~J}_{\mathrm{HH}} 7.0 \mathrm{~Hz}, \mathrm{CH}_{3}\right) .{ }^{13} \mathrm{C} \mathrm{NMR}\{1 \mathrm{H}\}\left(125 \mathrm{MHz} \mathrm{DMSO}-d_{6}\right) \delta(\mathrm{ppm}):$ $174.2(\mathrm{C}=\mathrm{S}), 173.5(\mathrm{C}=\mathrm{O}), 172.3(\mathrm{C}=\mathrm{O}), 167.5$ (C=O), 159.5, 158.3, 146.1, 128.3, 127.7, 83.4, 64.8, 63.6, 61.2, 41.0, 22.4, 21.4, 14.6, 14.1. Anal. Calcd for $\left(\mathrm{C}_{19} \mathrm{H}_{23} \mathrm{~N}_{3} \mathrm{O}_{6} \mathrm{~S}\right): \mathrm{C}, 54.14 ; \mathrm{H}, 5.50 ; \mathrm{N}, 9.97 ; \mathrm{S}, 7.61 \%$. Found: $\mathrm{C}, 54.22$; H, 5.68; N, 9.81; S,7.75\%.

5-(3-(4-(Dimethylamino)pyridin-1-ium-1-yl)-1,4-dimethoxy-1,4-dioxobutan-2-yl)-2,4,6-trioxohexahydro-

pyrimidin-5ide (4d). Yellow solid, $\mathrm{mp} 278-280{ }^{\circ} \mathrm{C}\left(90 \%\right.$ Yield); IR (KBr) $v_{\max } / \mathrm{cm}^{-1}: 3188,1692 .{ }^{1} \mathrm{H} \mathrm{NMR}(500$ MHz DMSO-d 6 ) $\delta$ (ppm): $10.33(1 \mathrm{H}, \mathrm{s}, \mathrm{NH}), 10.20(1 \mathrm{H}, \mathrm{s}, \mathrm{NH}), 8.16\left(2 \mathrm{H}, \mathrm{d},{ }^{3} J_{\mathrm{HH}} 5.8 \mathrm{~Hz}, \mathrm{H}-\mathrm{Ar}\right), 8.02\left(1 \mathrm{H}, \mathrm{d},{ }^{3} J_{\mathrm{HH}} 14.1\right.$ $\mathrm{Hz}, \mathrm{CH}), 7.34\left(1 \mathrm{H}, \mathrm{d},{ }^{3} J_{\mathrm{HH}} 14.1 \mathrm{~Hz}, \mathrm{CH}\right), 6.81\left(2 \mathrm{H}, \mathrm{d},{ }^{3} \mathrm{~J}_{\mathrm{HH}} 5.8 \mathrm{~Hz}, \mathrm{H}-\mathrm{Ar}\right), 3.31\left(3 \mathrm{H}, \mathrm{s}, \mathrm{CH}_{3}\right), 3.20\left(3 \mathrm{H}, \mathrm{s}, \mathrm{CH}_{3}\right), 3.08$ $\left(6 \mathrm{H}, \mathrm{s}, 2 \mathrm{CH}_{3}\right) .{ }^{13} \mathrm{C} N M R\{1 \mathrm{H}\}\left(125 \mathrm{MHz}\right.$ DMSO-d $\left.d_{6}\right) \delta(\mathrm{ppm}): 171.1(\mathrm{C}=0), 167.2(\mathrm{C}=0), 165.3(\mathrm{C}=0), 164.7(\mathrm{C}=0)$, $152.5,151.4,150.0,144.0,107.3,102.9,96.9,42.6,41.8,39.6$. Anal. Calcd for $\left(\mathrm{C}_{17} \mathrm{H}_{20} \mathrm{~N}_{4} \mathrm{O}_{7}\right): \mathrm{C}, 52.04 ; \mathrm{H}, 5.14$; N, $14.28 \%$. Found: C, 52.17; H, 5.06; N, 14.15\%.

5-(1,4-Dimethoxy-3-(2-methylquinolin-1-ium-1-yl)-1,4-dioxobutan-2-yl)-2,4,6-trioxohexahydropyrimidin-5-

ide 4e). Light brown solid, $\mathrm{mp} 177-180{ }^{\circ} \mathrm{C}\left(95 \%\right.$ Yield). IR $(\mathrm{KBr}) v_{\max } / \mathrm{cm}^{-1}: 3421,16955 .{ }^{1} \mathrm{H} \mathrm{NMR}(500 \mathrm{MHz}$ DMSO- $\left.d_{6}\right) \delta(\mathrm{ppm}): 11.77(1 \mathrm{H}, \mathrm{s}, \mathrm{NH}), 11.62(1 \mathrm{H}, \mathrm{s}, \mathrm{NH}), 7.05\left(1 \mathrm{H}, \mathrm{dd},{ }^{3} \mathrm{~J}_{\mathrm{HH}} 8.6,7.6 \mathrm{~Hz}, \mathrm{H}-\mathrm{Ar}\right), 6.89\left(1 \mathrm{H}, \mathrm{dd},{ }^{3} J_{\mathrm{HH}}\right.$ 7.6, $1.6 \mathrm{~Hz}, \mathrm{H}-\mathrm{Ar}), 6.59-6.57(1 \mathrm{H}, \mathrm{m}, \mathrm{H}-\mathrm{Ar}), 6.54\left(1 \mathrm{H}, \mathrm{d},{ }^{3} \mathrm{~J}_{\mathrm{HH}} 8.2 \mathrm{~Hz}, \mathrm{H}-\mathrm{Ar}\right), 6.48\left(1 \mathrm{H}, \mathrm{d},{ }^{3} \mathrm{~J}_{\mathrm{HH}} 10.0 \mathrm{~Hz}, \mathrm{H}-\mathrm{Ar}\right), 5.49$ $\left(1 \mathrm{H}, \mathrm{d},{ }^{3} \mathrm{~J}_{\mathrm{HH}} 10.0 \mathrm{~Hz}, \mathrm{H}-\mathrm{Ar}\right), 4.80\left(1 \mathrm{H}, \mathrm{d},{ }^{3} \mathrm{~J}_{\mathrm{HH}} 7.3 \mathrm{~Hz}, \mathrm{CH}\right), 4.49\left(1 \mathrm{H}, \mathrm{d},{ }^{3} \mathrm{~J}_{\mathrm{HH}} 7.3 \mathrm{~Hz}, \mathrm{H}-\mathrm{Ar}\right), 3.79\left(3 \mathrm{H}, \mathrm{s}, \mathrm{CH}_{3}\right), 3.58$ $\left(3 \mathrm{H}, \mathrm{s}, \mathrm{CH}_{3}\right), 1.29\left(3 \mathrm{H}, \mathrm{s}, \mathrm{CH}_{3}\right) \cdot{ }^{13} \mathrm{C} \mathrm{NMR}\{1 \mathrm{H}\}(125 \mathrm{MHz}$ DMSO-d 6$) \delta(\mathrm{ppm}): 173.5(\mathrm{C}=\mathrm{O}), 170.3(\mathrm{C}=\mathrm{O}), 170.1$ (C=O), 167.3 (C=O), 150.2, 142.8, 130.3, 127.6, 127.0, 121.1, 118.3, 118.0, 110.8, 73.0, 68.4, 64.2, 53.3, 48.5, 29.6. Anal. Calcd for $\left(\mathrm{C}_{20} \mathrm{H}_{19} \mathrm{~N}_{3} \mathrm{O}_{7}\right)$ : C, 58.11; $\mathrm{H}, 4.63 ; \mathrm{N}, 10.16 \%$. Found: $\mathrm{C}, 58.29 ; \mathrm{H}, 4.85 ; \mathrm{N}, 10.31 \%$.

5-(1,4-Diethoxy-1,4-dioxo-3-(pyridin-1-ium-1-yl)butan-2-yl)-4,6-dioxo-2-thioxohexahydropyrimidin-5-ide (4f). Yellow solid, mp 197-199 C (97\% Yield). IR (KBr) $v_{\max } / \mathrm{cm}^{-1}: 3419,2252,1619 .{ }^{1} \mathrm{H}$ NMR (500 MHz DMSO-d 6 ) $\delta$ (ppm) major isomer: $10.85(2 \mathrm{H}, \mathrm{bs}, 2 \mathrm{NH}), 8.89-8.85(2 \mathrm{H}, \mathrm{m}, \mathrm{H}-\mathrm{Ar}), 8.51-8.47(1 \mathrm{H}, \mathrm{m}, \mathrm{H}-\mathrm{Ar}), 7.98\left(2 \mathrm{H}, \mathrm{t},{ }^{3} J_{\mathrm{HH}}\right.$ $6.3 \mathrm{~Hz}, \mathrm{H}-\mathrm{Ar}), 6.23\left(1 \mathrm{H}, \mathrm{d},{ }^{3} J_{\mathrm{HH}} 9.1 \mathrm{~Hz}, \mathrm{CH}\right), 4.69\left(1 \mathrm{H}, \mathrm{d},{ }^{3} J_{\mathrm{HH}} 9.1 \mathrm{~Hz}, \mathrm{CH}\right), 4.05\left(2 \mathrm{H}, \mathrm{q}^{3}{ }^{3} \mathrm{JHH}_{\mathrm{HH}} 7.1 \mathrm{~Hz}, \mathrm{CH}_{2}\right), 3.94(2 \mathrm{H}$, $\left.q,{ }^{3} J_{\mathrm{HH}} 7.1 \mathrm{~Hz}, \mathrm{CH}_{2}\right), 1.21-1.10\left(6 \mathrm{H}, \mathrm{m}, 2 \mathrm{CH}_{3}\right) .{ }^{13} \mathrm{C} \mathrm{NMR}\{1 \mathrm{H}\}\left(125 \mathrm{MHz} \mathrm{DMSO}-d_{6}\right) \delta(\mathrm{ppm})$ major isomer: 174.2 $(C=S), 168.9$ (C=O), 167.1 ( $C=0), 162.1(C=0), 147.7,145.7,127.3,105.4,89.8,63.1,59.8,58.9,14.8,14.2 .{ }^{1} \mathrm{H}$ NMR $\left(500 \mathrm{MHz}\right.$ DMSO- $\left.d_{6}\right) \delta(\mathrm{ppm})$ minor isomer: $10.85(2 \mathrm{H}, \mathrm{bs}, 2 \mathrm{NH}), 8.91\left(2 \mathrm{H}, \mathrm{d},{ }^{3} \mathrm{~J}_{\mathrm{HH}} 7.3 \mathrm{~Hz}, \mathrm{H}-\mathrm{Ar}\right), 8.59(1 \mathrm{H}$, d, $\left.{ }^{3} J_{\mathrm{HH}} 8.0 \mathrm{~Hz}, \mathrm{H}-\mathrm{Ar}\right), 8.05\left(2 \mathrm{H}, \mathrm{t},{ }^{3} J_{\mathrm{HH}} 7.1 \mathrm{~Hz}, \mathrm{H}-\mathrm{Ar}\right), 5.84\left(1 \mathrm{H}, \mathrm{d},{ }^{3} J_{\mathrm{HH}} 6.3 \mathrm{~Hz}, \mathrm{CH}\right), 4.73\left(1 \mathrm{H}, \mathrm{d},{ }^{3} \mathrm{~J}_{\mathrm{HH}} 6.3 \mathrm{~Hz}, \mathrm{CH}\right)$, 4.26-4.15 $\left(4 \mathrm{H}, \mathrm{m}, 2 \mathrm{CH}_{2}\right), 1.21-1.10\left(6 \mathrm{H}, \mathrm{m}, 2 \mathrm{CH}_{3}\right) .{ }^{13} \mathrm{C} \mathrm{NMR}\{1 \mathrm{H}\}\left(125 \mathrm{MHz} \mathrm{DMSO}-d_{6}\right) \delta(\mathrm{ppm})$ minor isomer: $174.4(\mathrm{C}=\mathrm{S}), 171.6(\mathrm{C}=\mathrm{O}), 167.5(\mathrm{C}=\mathrm{O}), 162.7$ ( $\mathrm{C}=\mathrm{O}), 147.5,145.9,127.6,106.4,83.7,70.52,61.1,56.5,14.5$, 
14.1. Anal. Calcd for $\left(\mathrm{C}_{17} \mathrm{H}_{19} \mathrm{~N}_{3} \mathrm{O}_{6} \mathrm{~S}\right)$ : C, 51.90; $\mathrm{H}, 4.87 ; \mathrm{N}, 10.68 ; \mathrm{S}, 8.15 \%$. Found: $\mathrm{C}, 51.68 ; \mathrm{H}, 4.64 ; \mathrm{N}, 10.86 ; \mathrm{S}$, $8.31 \%$.

5-(1,4-Dimethoxy-3-(6-methylisoquinolin-2-ium-2-yl)-1,4-dioxobutan-2-yl)-2,4,6-trioxohexahydropyrimidin-

5-ide (4g). Light brown solid, $\mathrm{mp} 187-190{ }^{\circ} \mathrm{C}\left(96 \%\right.$ Yield). IR (KBr) $v_{\max } / \mathrm{cm}^{-1}: 3417,2254,1737,1650 .{ }^{1} \mathrm{H} \mathrm{NMR}$ (500 MHz DMSO- $\left.d_{6}\right) \delta(\mathrm{ppm}): 11.70(2 \mathrm{H}, \mathrm{bs}, 2 \mathrm{NH}), 11.10\left(1 \mathrm{H}, \mathrm{s}, \mathrm{H}-\mathrm{C}=\mathrm{N}^{+}\right), 6.89\left(1 \mathrm{H}, \mathrm{d}^{3}{ }^{3} \mathrm{HH}_{\mathrm{HH}} 8.1 \mathrm{~Hz}, \mathrm{H}-\mathrm{Ar}\right), 6.71$ $(1 \mathrm{H}, \mathrm{s}, \mathrm{H}-\mathrm{Ar}), 6.58\left(1 \mathrm{H}, \mathrm{d},{ }^{3} \mathrm{~J}_{\mathrm{HH}} 10.1 \mathrm{~Hz}, \mathrm{H}-\mathrm{Ar}\right), 6.49\left(1 \mathrm{H}, \mathrm{d},{ }^{3} J_{\mathrm{HH}} 8.1 \mathrm{~Hz}, \mathrm{H}-\mathrm{Ar}\right), 5.62\left(1 \mathrm{H}, \mathrm{d},{ }^{3} J_{\mathrm{HH}} 10.1 \mathrm{~Hz}, \mathrm{H}-\mathrm{Ar}\right), 4.83$ $\left(1 \mathrm{H}, \mathrm{d},{ }^{3} J_{\mathrm{HH}} 7.1 \mathrm{~Hz}, \mathrm{CH}\right), 4.35\left(1 \mathrm{H}, \mathrm{d},{ }^{3} \mathrm{~J}_{\mathrm{HH}} 7.1 \mathrm{~Hz}, \mathrm{CH}\right), 3.78\left(3 \mathrm{H}, \mathrm{s}, \mathrm{CH}_{3}\right), 3.59\left(3 \mathrm{H}, \mathrm{s}, \mathrm{CH}_{3}\right), 2.10\left(3 \mathrm{H}, \mathrm{s}, \mathrm{CH}_{3}\right) .{ }^{13} \mathrm{C}$ NMR $\{1 \mathrm{H}\}(125 \mathrm{MHz}$ DMSO-d $) \delta(\mathrm{ppm}): 172.5(\mathrm{C}=0), 169.7(\mathrm{C}=0), 168.4(\mathrm{C}=0), 164.1(\mathrm{C}=0), 152.1,150.2$, 141.1, 131.0, 130.5, 128.4, 118.8, 115.8, 110.1, 74.3, 65.7, 64.8, 53.4, 51.3, 20.2. Anal. Calcd for $\left(\mathrm{C}_{20} \mathrm{H}_{19} \mathrm{~N}_{3} \mathrm{O}_{7}\right)$ : C, 58.11; H, 4.63; N, 10.16\%. Found: C, 58.27; H, 4.50; N, $10.09 \%$.

5-(1,4-Diethoxy-3-(2-methylquinolin-1-ium-1-yl)-1,4-dioxobutan-2-yl)-4,6-dioxo-2-thioxohexahydro-

pyrimidin-5-ide (4h). Light brown solid, mp $194-196{ }^{\circ} \mathrm{C}\left(97 \%\right.$ Yield). IR (KBr) $v_{\max } / \mathrm{cm}^{-1}: 3419,1725 .{ }^{1} \mathrm{H} N M R$ (500 MHz DMSO- $\left.d_{6}\right) \delta(\mathrm{ppm})$ major isomer: $10.20(1 \mathrm{H}, \mathrm{s}, 2 \mathrm{NH}), 9.09\left(1 \mathrm{H}, \mathrm{d},{ }^{3} \mathrm{~J}_{\mathrm{HH}} 8.5 \mathrm{~Hz}, \mathrm{H}-\mathrm{Ar}\right), 8.27\left(1 \mathrm{H}, \mathrm{dd},{ }^{3} \mathrm{~J}_{\mathrm{HH}}\right.$ 8.2, $1.6 \mathrm{~Hz}, \mathrm{H}-\mathrm{Ar}), 8.06\left(1 \mathrm{H}, \mathrm{d},{ }^{3} \mathrm{~J}_{\mathrm{HH}} 8.5 \mathrm{~Hz}, \mathrm{H}-\mathrm{Ar}\right), 7.98\left(1 \mathrm{H}, \mathrm{ddd},{ }^{3} J_{\mathrm{HH}} 8.8,7.1,1.6 \mathrm{~Hz}, \mathrm{H}-\mathrm{Ar}\right), 7.83-7.80(1 \mathrm{H}, \mathrm{m}, \mathrm{H}-$ Ar), $7.78\left(1 \mathrm{H}, \mathrm{d},{ }^{3} \mathrm{~J}_{\mathrm{HH}} 8.8 \mathrm{~Hz}, \mathrm{H}-\mathrm{Ar}\right), 7.19\left(1 \mathrm{H}, \mathrm{d},{ }^{3} \mathrm{~J}_{\mathrm{HH}} 7.4 \mathrm{~Hz}, \mathrm{CH}\right), 5.10\left(1 \mathrm{H}, \mathrm{d},{ }^{3} J_{\mathrm{HH}} 7.4 \mathrm{~Hz}, \mathrm{CH}\right), 4.17-4.02(4 \mathrm{H}, \mathrm{m}$, $\left.2 \mathrm{CH}_{2}\right), 3.37\left(3 \mathrm{H}, \mathrm{s}, \mathrm{CH}_{3}\right), 1.11\left(3 \mathrm{H}, \mathrm{t},{ }^{3} J_{\mathrm{HH}} 7.1 \mathrm{~Hz}, \mathrm{CH}_{3}\right), 0.93\left(3 \mathrm{H}, \mathrm{t},{ }^{3} \mathrm{JHH}_{\mathrm{HH}} 7.1 \mathrm{~Hz}, 3 \mathrm{H}, \mathrm{CH}_{3}\right) .{ }^{13} \mathrm{C} \mathrm{NMR}\{1 \mathrm{H}\}(125 \mathrm{MHz}$ DMSO- $\left.d_{6}\right) \delta(\mathrm{ppm})$ major isomer: $173.8(\mathrm{C}=\mathrm{S}), 172.9(\mathrm{C}=\mathrm{O}), 168.1(\mathrm{C}=0), 164.8(\mathrm{C}=\mathrm{O}), 148.1,139.5,135.3$, $130.8,129.2,127.8,125.4,119.8,84.5,64.4,63.3,61.3,41.8,23.9,14.6,14.0$. Anal. Calcd for $\left(\mathrm{C}_{22} \mathrm{H}_{23} \mathrm{~N}_{3} \mathrm{O}_{6} \mathrm{~S}\right): \mathrm{C}$, 57.76; H, 5.07; N, 9.18; S, 7.01\%. Found: C, 57.49; H, 5.13; N, 9.32; S, 7.17\%.

${ }^{1} \mathrm{H}$ NMR $(500 \mathrm{MHz}$ DMSO-d $) \delta(\mathrm{ppm})$ minor isomer: $12.07(1 \mathrm{H}, \mathrm{s}, \mathrm{NH}), 11.91(1 \mathrm{H}, \mathrm{s}, \mathrm{NH}), 8.06\left(1 \mathrm{H}, \mathrm{d},{ }^{3} \mathrm{~J} \mathrm{HH} 8.5\right.$ $\mathrm{Hz}, \mathrm{H}-\mathrm{Ar}$ ), 7.95-7.86 (1H, m, H-Ar), 7.40 (1H, ddd, $\left.{ }^{3} \mathrm{H}_{\mathrm{HH}} 8.6,7.3,1.6 \mathrm{~Hz}, \mathrm{H}-\mathrm{Ar}\right), 7.36-7.31$ (1H, m, H-Ar), 7.23 (1H, d, $\left.{ }^{3} J_{н H} 7.0 \mathrm{~Hz}, \mathrm{H}-\mathrm{Ar}\right), 7.00\left(1 \mathrm{H}, \mathrm{t},{ }^{3} \mathrm{~J}_{\mathrm{HH}} 7.4 \mathrm{~Hz}, \mathrm{H}-\mathrm{Ar}\right), 5.64\left(1 \mathrm{H}, \mathrm{d},{ }^{3} \mathrm{~J}_{\mathrm{HH}} 8.0 \mathrm{~Hz}, \mathrm{CH}\right), 4.58\left(1 \mathrm{H}, \mathrm{d}^{3}{ }^{3} \mathrm{HH}^{8} 8.0 \mathrm{~Hz}, \mathrm{CH}\right)$, $4.23\left(2 \mathrm{H}, \mathrm{q},{ }^{3} \mathrm{~J}_{\mathrm{HH}} 7.1 \mathrm{~Hz}, \mathrm{CH}_{2}\right), 4.17-4.02\left(2 \mathrm{H}, \mathrm{m}, 2 \mathrm{H}, \mathrm{CH}_{2}\right), 2.47\left(3 \mathrm{H}, \mathrm{s}, \mathrm{CH}_{3}\right), 1.18\left(3 \mathrm{H}, \mathrm{t},{ }^{3} \mathrm{~J} \mathrm{HH} 7.1 \mathrm{~Hz}, \mathrm{CH}_{3}\right), 1.07$ $\left(3 \mathrm{H}, \mathrm{t},{ }^{3} \mathrm{JHH}_{\mathrm{H}} 7.1 \mathrm{~Hz}, \mathrm{CH}_{3}\right) .{ }^{13} \mathrm{C} \mathrm{NMR}\{1 \mathrm{H}\}\left(125 \mathrm{MHz} \mathrm{DMSO}-d_{6}\right) \delta(\mathrm{ppm})$ minor isomer: $1738(\mathrm{C}=\mathrm{S}), 1712(\mathrm{C}=\mathrm{O})$, 1622 (C=O), 159.6, 149.9, 142.3, 135.3, 132.0, 130.0, 128.7, 121.7, 113.8, 101.4, 64.2, 62.6, 61.7, 46.4, 27.7, $14.3,14.2$.

5-(1,4-Dimethoxy-1,4-dioxo-3-(quinolin-1-ium-1-yl)butan-2-yl)-2,4,6-trioxohexahydropyrimidin-5-ide (4i). Yellow solid, $\mathrm{mp} 201-203{ }^{\circ} \mathrm{C}$ (95\% Yield). IR ( KBr) $v_{\max } / \mathrm{cm}^{-1}: 3417,2254,1691 .{ }^{1} \mathrm{H} \mathrm{NMR}\left(500 \mathrm{MHz} \mathrm{DMSO}-d_{6}\right) \delta$ (ppm) major isomer: $10.07(1 \mathrm{H}, \mathrm{bs}, \mathrm{NH}), 10.02(1 \mathrm{H}, \mathrm{bs}, \mathrm{NH}), 9.37(1 \mathrm{H}, \mathrm{bs}, \mathrm{H}-\mathrm{Ar}), 8.52\left(1 \mathrm{H}, \mathrm{d},{ }^{3} \mathrm{~J} \mathrm{HH} 8.3 \mathrm{~Hz}, \mathrm{H}-\mathrm{Ar}\right)$, $8.42\left(1 \mathrm{H}, \mathrm{d},{ }^{3} \mathrm{~J}_{\mathrm{HH}} 7.0 \mathrm{~Hz}, \mathrm{H}-\mathrm{Ar}\right), 8.26\left(1 \mathrm{H}, \mathrm{d},{ }^{3} \mathrm{~J}_{\mathrm{HH}} 8.3 \mathrm{~Hz}, \mathrm{H}-\mathrm{Ar}\right), 8.22-8.17(1 \mathrm{H}, \mathrm{m}, \mathrm{H}-\mathrm{Ar}), 8.05-7.93(1 \mathrm{H}, \mathrm{m}, \mathrm{H}-\mathrm{Ar})$, 7.90-7.80 (1H, m, H-Ar), $5.92(1 \mathrm{H}, \mathrm{bs}, \mathrm{CH}), 4.88\left(1 \mathrm{H}, \mathrm{d},{ }^{3} \mathrm{~J}_{\mathrm{HH}} 6.3 \mathrm{~Hz}, \mathrm{CH}\right), 3.78\left(3 \mathrm{H}, \mathrm{s}, \mathrm{CH}_{3}\right), 3.57\left(3 \mathrm{H}, \mathrm{s}, \mathrm{CH}_{3}\right) .{ }^{13} \mathrm{C}$ NMR $\{1 \mathrm{H}\}\left(125 \mathrm{MHz}\right.$ DMSO- $\left.d_{6}\right) \delta(\mathrm{ppm})$ major isomer: $170.0(\mathrm{C}=0), 167.9(\mathrm{C}=0), 164.3(\mathrm{C}=0), 151.9(\mathrm{C}=0)$, 149.0, 148.9, 137.9, 135.5, 134.8, 130.4, 130.2, 127.6, 124.4, 102.2, 86.8, 56.5, 51.4, 50.4. ${ }^{1} \mathrm{H} \mathrm{NMR}(500 \mathrm{MHz}$ DMSO- $\left.d_{6}\right) \delta(\mathrm{ppm})$ minor isomer: $9.64(1 \mathrm{H}, \mathrm{bs}, \mathrm{NH}), 9.63(1 \mathrm{H}, \mathrm{bs}, \mathrm{NH}), 9.37(1 \mathrm{H}, \mathrm{bs}, \mathrm{H}-\mathrm{Ar}), 8.58\left(1 \mathrm{H}, \mathrm{d},{ }^{3} J_{\mathrm{HH}} 7.5\right.$ $\mathrm{Hz}, \mathrm{H}-\mathrm{Ar}), 8.34\left(1 \mathrm{H}, \mathrm{d},{ }^{3} \mathrm{~J}_{\mathrm{HH}} 8.3 \mathrm{~Hz}, \mathrm{H}-\mathrm{Ar}\right), 8.15\left(1 \mathrm{H}, \mathrm{d},{ }^{3} \mathrm{~J}_{\mathrm{HH}} 8.3 \mathrm{~Hz}, \mathrm{H}-\mathrm{Ar}\right), 8.05-7.93(1 \mathrm{H}, \mathrm{m}, \mathrm{H}-\mathrm{Ar}), 7.90-7.80(1 \mathrm{H}$, m, H-Ar), $7.67\left(1 \mathrm{H}, \mathrm{t},{ }^{3} \mathrm{~J}_{\mathrm{HH}} 7.7 \mathrm{~Hz}, \mathrm{H}-\mathrm{Ar}\right), 5.92(1 \mathrm{H}, \mathrm{bs}, \mathrm{CH}), 4.67\left(1 \mathrm{H}, \mathrm{d},{ }^{3} \mathrm{~J}_{\mathrm{HH}} 8.4 \mathrm{~Hz}, \mathrm{CH}\right), 3.76\left(3 \mathrm{H}, \mathrm{s}, \mathrm{CH}_{3}\right), 3.56$ $\left(1 \mathrm{H}, \mathrm{s}, \mathrm{CH}_{3}\right) .{ }^{13} \mathrm{C} N M R\{1 \mathrm{H}\}\left(125 \mathrm{MHz} \mathrm{DMSO}-d_{6}\right) \delta(\mathrm{ppm})$ minor isomer: $169.3(\mathrm{C}=0), 165.9(\mathrm{C}=0), 164.3(\mathrm{C}=0)$, 152.9 (C=O), 151.4, 151.3, 137.4, 135.5, 134.8, 131.6, 131.3, 127.7, 126.7, 92.7, 69.6, 53.8, 52.6, 44.4. Anal. Calcd for $\left(\mathrm{C}_{19} \mathrm{H}_{17} \mathrm{~N}_{3} \mathrm{O}_{7}\right): \mathrm{C}, 57.14 ; \mathrm{H}, 4.29 ; \mathrm{N}, 10.52 \%$. Found: $\mathrm{C}, 57.29 ; \mathrm{H}, 4.40 ; \mathrm{N}, 10.27 \%$.

5-(1,4-Dimethoxy-1,4-dioxo-3-(pyridin-1-ium-1-yl)butan-2-yl)-2,4,6-trioxohexahydropyrimidin-5-ide (4j). Yellow solid, mp $210-211{ }^{\circ} \mathrm{C}\left(98 \%\right.$ Yield). IR (KBr) $v_{\max } / \mathrm{cm}^{-1}: 3418,2253,1715 .{ }^{1} \mathrm{H}$ NMR (500 MHz DMSO-d 6 ) $\delta$ (ppm) major isomer: $11.10(2 \mathrm{H}, \mathrm{bs}, 2 \mathrm{NH}), 9.17\left(2 \mathrm{H}, \mathrm{d},{ }^{3} \mathrm{~J}_{\mathrm{HH}} 9.3 \mathrm{~Hz}, \mathrm{H}-\mathrm{Ar}\right), 8.55\left(1 \mathrm{H}, \mathrm{t},{ }^{3} \mathrm{~J}_{\mathrm{HH}} 8.0 \mathrm{~Hz}, \mathrm{H}-\mathrm{Ar}\right), 8.10-7.94$ $(2 \mathrm{H}, \mathrm{m}, \mathrm{H}-\mathrm{Ar}), 5.76\left(1 \mathrm{H}, \mathrm{d},{ }^{3} \mathrm{~J}_{\mathrm{HH}} 6.0 \mathrm{~Hz}, \mathrm{CH}\right), 4.73\left(1 \mathrm{H}, \mathrm{d},{ }^{3} \mathrm{~J}_{\mathrm{HH}} 6.0 \mathrm{~Hz}, \mathrm{CH}\right), 3.77\left(3 \mathrm{H}, \mathrm{s}, \mathrm{CH}_{3}\right), 3.54\left(3 \mathrm{H}, \mathrm{s}, \mathrm{CH}_{3}\right) .{ }^{13} \mathrm{C}$ NMR $\{1 \mathrm{H}\}\left(125 \mathrm{MHz}\right.$ DMSO- $\left.d_{6}\right) \delta(\mathrm{ppm})$ major isomer: $169.9(\mathrm{C}=0), 168.2(\mathrm{C}=0), 167.8(\mathrm{C}=0), 164.2(\mathrm{C}=0)$, 
152.1, 145.8, 126.3, 101.8, 72.1, 51.3, 50.4, 39.9. ${ }^{1} \mathrm{H}$ NMR (500 MHz DMSO- $\left.d_{6}\right) \delta(p p m)$ minor isomer 11.10 $(2 \mathrm{H}, \mathrm{bs}, 2 \mathrm{NH}), 8.97(2 \mathrm{H}, \mathrm{bs}, \mathrm{H}-\mathrm{Ar}), 8.84\left(1 \mathrm{H}, \mathrm{d},{ }^{3} \mathrm{~J}_{\mathrm{HH}} 6.0 \mathrm{~Hz}, \mathrm{H}-\mathrm{Ar}\right), 8.05-7.99(2 \mathrm{H}, \mathrm{m}, \mathrm{H}-\mathrm{Ar}), 6.24\left(1 \mathrm{H}, \mathrm{d},{ }^{3} \mathrm{~J}_{\mathrm{HH}} 8.6 \mathrm{~Hz}\right.$, $\mathrm{CH}), 4.67\left(1 \mathrm{H}, \mathrm{d},{ }^{3} J_{\mathrm{HH}} 8.6 \mathrm{~Hz}, \mathrm{CH}\right), 3.74\left(3 \mathrm{H}, \mathrm{s}, \mathrm{CH}_{3}\right), 3.54\left(3 \mathrm{H}, \mathrm{s}, \mathrm{CH}_{3}\right) .{ }^{13} \mathrm{C} \mathrm{NMR}\{1 \mathrm{H}\}\left(125 \mathrm{MHz} \mathrm{DMSO}-d_{6}\right) \delta(\mathrm{ppm})$ minor isomer: $169.9(\mathrm{C}=\mathrm{O}), 168.2(\mathrm{C}=\mathrm{O}), 167.8(\mathrm{C}=\mathrm{O}), 164.2(\mathrm{C}=\mathrm{O}), 151.3,147.5,126.8,87.7,70.6,53.7,52.5$, 44.6. Anal. Calcd for $\left(\mathrm{C}_{15} \mathrm{H}_{15} \mathrm{~N}_{3} \mathrm{O}_{7}\right)$ : C, 51.58; $\mathrm{H}, 4.33 ; \mathrm{N}, 12.03 \%$. Found: $\mathrm{C}, 51.35 ; \mathrm{H}, 4.26 ; \mathrm{N}, 11.89 \%$.

5-(1,4-Diethoxy-3-(isoquinolin-2-ium-2-yl)-1,4-dioxobutan-2-yl)-2,4,6-trioxohexahydropyrimidin-5-ide (4k). Brown solid, $\mathrm{mp} 197-200{ }^{\circ} \mathrm{C}\left(94 \%\right.$ Yield). IR ( KBr) $v_{\max } / \mathrm{cm}^{-1}: 3422,1687 .{ }^{1} \mathrm{H}$ NMR (500 MHz DMSO-d $) \delta(p p m)$ major isomer: $10.31(2 \mathrm{H}, \mathrm{bs}, 2 \mathrm{NH}), 9.08\left(1 \mathrm{H}, \mathrm{s}, \mathrm{H}-\mathrm{C}=\mathrm{N}^{+}\right), 8.64(1 \mathrm{H}, \mathrm{bs}, \mathrm{H}-\mathrm{Ar}), 8.51\left(1 \mathrm{H}, \mathrm{d}^{3}{ }^{3} \mathrm{HH}_{\mathrm{H}} 8.3 \mathrm{~Hz}, \mathrm{H}-\mathrm{Ar}\right), 8.40$ $\left(1 \mathrm{H}, \mathrm{d},{ }^{3} \mathrm{~J}_{\mathrm{HH}} 6.9 \mathrm{~Hz}, \mathrm{H}-\mathrm{Ar}\right), 8.27\left(1 \mathrm{H}, \mathrm{d},{ }^{3} \mathrm{~J}_{\mathrm{HH}} 8.2 \mathrm{~Hz}, \mathrm{H}-\mathrm{Ar}\right), 8.22\left(1 \mathrm{H}, \mathrm{t},{ }^{3} \mathrm{~J}_{\mathrm{HH}} 7.4 \mathrm{~Hz}, \mathrm{H}-\mathrm{Ar}\right), 7.99\left(1 \mathrm{H}, \mathrm{t},{ }^{3} J_{\mathrm{HH}} 8.2 \mathrm{~Hz}, \mathrm{H}-\right.$ $\mathrm{Ar}), 5.88\left(1 \mathrm{H}, \mathrm{d},{ }^{3} \mathrm{~J}_{\mathrm{HH}} 6.4 \mathrm{~Hz}, \mathrm{CH}\right), 4.81\left(1 \mathrm{H}, \mathrm{d},{ }^{3} \mathrm{~J}_{\mathrm{HH}} 6.4 \mathrm{~Hz}, \mathrm{CH}\right), 4.29-4.20\left(2 \mathrm{H}, \mathrm{m}, \mathrm{CH}_{2}\right), 4.06-3.98\left(2 \mathrm{H}, \mathrm{m}, \mathrm{CH}_{2}\right)$, 1.24-1.22 $\left(3 \mathrm{H}, \mathrm{m}, \mathrm{CH}_{3}\right), 1.11-1.09\left(3 \mathrm{H}, \mathrm{m}, \mathrm{CH}_{3}\right) .{ }^{13} \mathrm{C} \mathrm{NMR}\{1 \mathrm{H}\}\left(125 \mathrm{MHz} \mathrm{DMSO}-d_{6}\right) \delta$ (ppm) major isomer: 173.8 $(\mathrm{C}=0), 169.6$ ( $\mathrm{C}=0), 167.6$ (C=O), 164.3 ( $\mathrm{C}=\mathrm{O}), 152.9,152.2,137.8,137.5,131.6,131.3,127.5,126.6,123.9$, 96.3, 72.1, 62.6, 61.0, 45.0, 14.5, 14.3. ${ }^{1} \mathrm{H}$ NMR $\left(500 \mathrm{MHz}\right.$ DMSO- $\left.d_{6}\right) \delta$ (ppm) minor isomer: 10.09 (2H, bs, 2NH), $9.08(1 \mathrm{H}, \mathrm{s}, \mathrm{H}-\mathrm{Ar}), 7.71\left(1 \mathrm{H}, \mathrm{d},{ }^{3} \mathrm{~J}_{\mathrm{HH}} 7.5 \mathrm{~Hz}, \mathrm{H}-\mathrm{Ar}\right), 7.62-7.59(3 \mathrm{H}, \mathrm{m}, \mathrm{H}-\mathrm{Ar}), 8.49\left(1 \mathrm{H}, \mathrm{t},{ }^{3} \mathrm{~J}_{\mathrm{HH}} 7.5 \mathrm{~Hz}, \mathrm{H}-\mathrm{Ar}\right)$, $7.45(1 \mathrm{H}, \mathrm{bs}, \mathrm{H}-\mathrm{Ar}), 5.57\left(1 \mathrm{H}, \mathrm{d},{ }^{3} \mathrm{~J}_{\mathrm{HH}} 8.1 \mathrm{~Hz}, \mathrm{CH}\right), 4.50\left(1 \mathrm{H}, \mathrm{d}^{3}{ }^{3} \mathrm{HHH}_{\mathrm{HH}} 8.1 \mathrm{~Hz}, \mathrm{CH}\right), 4.29-4.20\left(2 \mathrm{H}, \mathrm{m}, \mathrm{CH}_{2}\right), 4.06-3.98$ $\left(2 \mathrm{H}, \mathrm{m}, \mathrm{CH}_{2}\right), 1.24-1.22\left(3 \mathrm{H}, \mathrm{m}, \mathrm{CH}_{3}\right), 1.11-1.09\left(3 \mathrm{H}, \mathrm{m}, \mathrm{CH}_{3}\right) .{ }^{13} \mathrm{C} \mathrm{NMR}\{1 \mathrm{H}\}\left(125 \mathrm{MHz} \mathrm{DMSO}-d_{6}\right) \delta(\mathrm{ppm}) \mathrm{minor}$ isomer: $170.7(\mathrm{C}=\mathrm{O}), 167.6(\mathrm{C}=\mathrm{O}), 167.0(\mathrm{C}=\mathrm{O}), 164.2(\mathrm{C}=\mathrm{O}), 151.0,137.8,136.6,135.3,134.0,128.7,128.5$, $126.3,125.3,79.3,72.99,69.2,62.53,61.4,14.3,14.3$. Anal. Calcd for $\left(\mathrm{C}_{21} \mathrm{H}_{21} \mathrm{~N}_{3} \mathrm{O}_{7}\right): \mathrm{C}, 59.01 ; \mathrm{H}, 4.95 ; \mathrm{N}, 9.83 \%$. Found: C, 59.20; $\mathrm{H}, 4.81 ; \mathrm{N}, 9.96 \%$.

5-(3-(Isoquinolin-2-ium-2-yl)-1,4-dimethoxy-1,4-dioxobutan-2-yl)-4,6-dioxo-2-thioxohexahydropyrimidin-5ide (4l). Light brown solid, mp 190-193 ${ }^{\circ} \mathrm{C}$ (92\% Yield). ${ }^{1} \mathrm{H}$ NMR (500 MHz DMSO- $\left.d_{6}\right) \delta(p p m)$ major isomer: $10.60(2 \mathrm{H}, \mathrm{s}, 2 \mathrm{NH}), 10.10\left(1 \mathrm{H}, \mathrm{s}, \mathrm{H}-\mathrm{C}=\mathrm{N}^{+}\right), 8.57-8.54(2 \mathrm{H}, \mathrm{m}, \mathrm{H}-\mathrm{Ar}), 8.45-8.44(1 \mathrm{H}, \mathrm{m}, \mathrm{H}-\mathrm{Ar}), 8.30-8.21(2 \mathrm{H}, \mathrm{m}, \mathrm{H}-$ $\mathrm{Ar})$, 8.07-7.99 (1H, m, H-Ar), $6.31\left(1 \mathrm{H}, \mathrm{d},{ }^{3} \mathrm{~J}_{\mathrm{HH}} 9.3 \mathrm{~Hz}, \mathrm{CH}\right), 4.82\left(1 \mathrm{H}, \mathrm{d},{ }^{3} \mathrm{~J}_{\mathrm{HH}} 9.3 \mathrm{~Hz}, \mathrm{CH}\right), 3.75\left(3 \mathrm{H}, \mathrm{s}, \mathrm{CH}_{3}\right), 3.57$ $\left(3 \mathrm{H}, \mathrm{s}, \mathrm{CH}_{3}\right) .{ }^{13} \mathrm{C} N M R\{1 \mathrm{H}\}(125 \mathrm{MHz}$ DMSO-d $) \delta(\mathrm{ppm})$ major isomer: $174.4(\mathrm{C}=\mathrm{S}), 173.9(\mathrm{C}=0), 168.1(\mathrm{C}=\mathrm{O})$, 162.7 (C=O), 151.3, 138.1, 137.4, 131.7, 128.9, 127.7, 127.2, 125.0, 122.3, 83.7, 70.4, 54.1, 52.5, 43.1. ${ }^{1} \mathrm{H}-\mathrm{NMR}$ (500 MHz DMSO- $\left.d_{6}\right): \delta(p p m)$ minor isomer: $10.56(2 \mathrm{H}, \mathrm{s}, 2 \mathrm{NH}), 9.98(1 \mathrm{H}, \mathrm{s}, \mathrm{H}-\mathrm{Ar}), 8.68-8.60(1 \mathrm{H}, \mathrm{m}, \mathrm{H}-\mathrm{Ar})$, $8.49\left(1 \mathrm{H}, \mathrm{d},{ }^{3} \mathrm{~J}_{\mathrm{HH}} 8.3 \mathrm{~Hz}, \mathrm{H}-\mathrm{Ar}\right), 8.07-8.05(1 \mathrm{H}, \mathrm{m}, \mathrm{H}-\mathrm{Ar}), 8.03-7.99(1 \mathrm{H}, \mathrm{m}, \mathrm{H}-\mathrm{Ar}), 7.92-7.88(1 \mathrm{H}, \mathrm{m}, \mathrm{H}-\mathrm{Ar}), 7.80-$ $7.76(1 \mathrm{H}, \mathrm{m}, \mathrm{H}-\mathrm{Ar}), 5.94\left(1 \mathrm{H}, \mathrm{d},{ }^{3}{ }_{\mathrm{HH}} 6.2 \mathrm{~Hz}, \mathrm{CH}\right), 4.85\left(1 \mathrm{H}, \mathrm{d}^{3}{ }^{3} \mathrm{~J}_{\mathrm{HH}} 6.2 \mathrm{~Hz}, \mathrm{CH}\right), 3.79\left(3 \mathrm{H}, \mathrm{s}, \mathrm{CH}_{3}\right), 3.58\left(3 \mathrm{H}, \mathrm{s}, \mathrm{CH}_{3}\right)$. ${ }^{13} \mathrm{C} \mathrm{NMR}\{1 \mathrm{H}\}\left(125 \mathrm{MHz} \mathrm{DMSO}-d_{6}\right) \delta(\mathrm{ppm})$ minor isomer: $174.4(\mathrm{C}=\mathrm{S}), 173.4(\mathrm{C}=0), 167.9(\mathrm{C}=0), 162.5(\mathrm{C}=0)$, 153.0, 138.0, 132.9, 131.4, 128.3, 127.5, 126.9, 126.7, 124.3, 83.1, 70.7, 53.8, 52.7, 44.3. Anal. Calcd for $\left(\mathrm{C}_{19} \mathrm{H}_{17} \mathrm{~N}_{3} \mathrm{O}_{6} \mathrm{~S}\right): \mathrm{C}, 54.93 ; \mathrm{H}, 4.12 ; \mathrm{N}, 10.12 ; \mathrm{S}, 7.72 \%$. Found: $\mathrm{C}, 54.80 ; \mathrm{H}, 4.21 ; \mathrm{N}, 10.26 ; \mathrm{S}, 7.59 \%$.

\section{Acknowledgements}

We gratefully acknowledge a Vail-e-Asr University of Rafsanjan Faculty Research Grant for financial support

\section{Supplementary Material}

The experimental procedures and IR, ${ }^{1} \mathrm{H}$ NMR and ${ }^{13} \mathrm{C}$ NMR spectra associated with this article are available as supplementary data. 


\section{References}

1. Rathee, P.; Tonk, R. K.; Dalal, A.; Ruhil, M. K.; Kumar, A. Org. Cell. Mol. Biol. 2016, 62, 5.

2. Mobinikhaledi, A.; Kalhor, M. Int. J . Drug Dev. Res. 2010, 2, 268-272.

3. Mohamed, N. R.; El-Saidi, M. M. T.; Ali, Y. M.; Elnagdi, M. H. Bioorg. Med. Chem. 2007, 15, 6227-6735. https://doi.org/10.1016/j.bmc.2007.06.023

4. Bartzatt, R. J . Pharm. Biomed. Anal. 2002, 29, 909-915. https://doi.org/10.1016/S0731-7085(02)00168-1

5. McClenaghan, N. D.; Absalon, C.; Bassani, D. M. J . Am. Chem. Soc. 2003, 125, 13004-13005. https://doi.org/10.1021/ja0372098

6. Look, S. A.; Burch, M. T.; Fenical, W.; Qi-tai, Z.; Clardy, J . J. Org. Chem. 1985, 50, 5741-5746. https://doi.org/10.1021/jo00350a061

7. Litvinov, V. P.; Shestopalov, A. M. Russ. J. Org. Chem. 1997, 33, 903-940.

8. Yavari, I.; Mokhtarporyani-Sanandaj, A.; Moradi, M.; Mirzaei, A. Tetrahedron 2008, 64, 5221-5225. https://doi.org/10.1016/j.tet.2008.03.044

9. Balas, V. I.; Hadjikakou, S. K.; Hadjiliadis, N.; Kourkoumelis, N.; Light, M. E.; Hursthouse, M.; Metsios, A. K.; Karkabounas, S. Bioinorg. Chem. Appl. 2008, 654137. https://doi.org/10.1155/2008/654137

10. Visser, P.; Zuhse, R.; Wong, M. W.; Wentrup, C. J. Am. Chem. Soc. 1996, 118, 12598-12602. https://doi.org/10.1021/ja9626720

11. Kuhn, A.; Plüg, C.; Wentrup, C. J. Am. Chem. Soc. 2000, 122, 1945-1948. https://doi.org/10.1021/ja993859t

12. Rudler, H.; Durand-Réville, T. J. Organometal. Chem. 2001, 617-618, 571-587. https://doi.org/10.1016/S0022-328X(00)00727-0

13. Yavari, I.; Anary-Abbasinejad, M.; Alizadeh, A. Monatsh. Chem. 2002, 133, 1331-1336. https://doi.org/10.1007/s007060200110

14. Xia, E. Y.; Sun, J.; Yao, R.; Yan, C. G. Tetrahedron 2010, 66, 3569-3574. https://doi.org/10.1016/i.tet.2010.03.060

15. Shaabani, A.; Bazgir, A.; Tavasoli-Rad, F.; Bijanzadeh, H. R.; Razmara, F. J. Chem. Res. 2002, 133-134.

16. Pourshamsian, K. ; Montazeri, N. ; Ali-Asgari, S. ; Zeydi, M. M. Biazar, E. Orient. J. Chem. 2011, 27, 10171023.

17. Han, Y.; Chen, J .; Hui, L.; Yan, C. G. Tetrahedron 2010, 66, 7743-7748. https://doi.org/10.1016/i.tet.2010.07.073

18. Wang, Q. F.; Hui, L.; Hou, H.; Yan, C. G. J . Comb. Chem. 2010, 12, 260-265. https://doi.org/10.1021/cc900161z

19. J in, G., Sun, J . and Yan, C. G. RSC Adv. 2016, 87, 84379-84387.

20. Kumar, A.; Gupta, G.; Srivastava, S. Org. Lett. 2011, 13, 6366-6369. https://doi.org/10.1021/ol202654j

21. Yavari, I.; Anary-Abbasinejad, M.; Alizadeh, A. S. Phosphorus Sulfur Silicon Rel. Elem. 2002, 177, 93-103. https://doi.org/10.1080/10426500210216 